Meta

Journal des tradlucteurs

Translators' Journal

\title{
Les horaires variables
}

\section{Viviane Forest et Manon Roy}

Volume 21, numéro 3, septembre 1976

URI : https://id.erudit.org/iderudit/002758ar

DOI : https://doi.org/10.7202/002758ar

Aller au sommaire du numéro

Éditeur(s)

Les Presses de l'Université de Montréal

ISSN

0026-0452 (imprimé)

1492-1421 (numérique)

Découvrir la revue

Citer cet article

Forest, V. \& Roy, M. (1976). Les horaires variables. Meta, 21(3), 205-210.

https://doi.org/10.7202/002758ar

Ce document est protégé par la loi sur le droit d'auteur. L’utilisation des services d'Érudit (y compris la reproduction) est assujettie à sa politique d'utilisation que vous pouvez consulter en ligne.

https://apropos.erudit.org/fr/usagers/politique-dutilisation/
Cet article est diffusé et préservé par Érudit.

Érudit est un consortium interuniversitaire sans but lucratif composé de l’Université de Montréal, l'Université Laval et l'Université du Québec à Montréal. Il a pour mission la promotion et la valorisation de la recherche. https://www.erudit.org/fr/ 


\section{LES HORAIRES VARIABLES}

Depuis 1960 , sous l'impulsion de la Suisse et de l'Allemagne, une nouvelle forme d'horaire de travail, l'horaire variable, s'est répandu au sein des entreprises privées et des gouvernements, marquant une étape supplémentaire vers une plus grande humanisation du travail. Ce concept a donné naissance à une terminologie française riche mais encore incertaine. En effet, on parle indifféremment d'horaires libres, flexibles, mobiles, variables, individuels, flottants, dynamiques, « à la carte », etc. L'OCDE nous donne la raison de cette pléthore de termes : "Son appellation n'est pas uniforme parce qu'elle est issue de la pratique des entreprises. »

Afin de faire de la lumière sur le sujet, nous avons donc commencé par délimiter les notions à l'aide de l'anglais, dont le vocabulaire est plus uniforme dans ce domaine. Nous nous sommes alors rendu compte que la différenciation de ces concepts repose sur trois variables : la source (l'horaire est imposé par la direction ou choisi par l'employé), l'application (l'horaire est applicable à tout le personnel,

\section{CLASSIFICATION DES HORAIRES VARIABLES PAR ORDRE CROISSANT DU DEGRÉ DE LIBERTÉ LAISSÉ À L'EMPLOYÉ}

\begin{tabular}{|c|c|c|c|c|}
\hline $\mathbf{N}^{\circ}$ & Termes anglais et français & Source & Application & Temps \\
\hline 1. & $\begin{array}{l}\text { fixed working hours } \\
\text { horaire fixe }\end{array}$ & horaire imposé & collectif & durable \\
\hline 2. & $\begin{array}{l}\text { staggered day work } \\
\text { horaire échelonné }\end{array}$ & horaire imposé & par groupes & durable \\
\hline $3 a$. & $\begin{array}{l}\text { staggered working hours } \\
\text { horaire décalé }\end{array}$ & nouvel horaire imposé & collectif & durable \\
\hline $3 b$. & $\begin{array}{l}\text { staggered working hours } \\
\text { horaire au choix }\end{array}$ & $\begin{array}{l}\text { choix partiellement } \\
\text { libre ou dirigé }\end{array}$ & collectif & durable \\
\hline $4 a$. & $\begin{array}{l}\text { individual working time } \\
\text { horaire individuel }\end{array}$ & choix dirigé & individuel & durable \\
\hline $4 b$. & $\begin{array}{l}\text { fixed flexible working hours } \\
\text { horaire varié }\end{array}$ & $\begin{array}{l}\text { choix partiellement } \\
\text { libre }\end{array}$ & individuel & durable \\
\hline $5 a$. & $\begin{array}{l}\text { flexible working hours }(\mathrm{G}) \\
\text { horaire variable }(\mathrm{G})\end{array}$ & choix assez libre & individuel & quotidien \\
\hline $5 b$. & $\begin{array}{l}\text { flexible working day (S) } \\
\text { horaire souple (S) }\end{array}$ & $\begin{array}{l}\text { choix partiellement } \\
\text { libre }\end{array}$ & individuel & quotidien \\
\hline $5 c$. & $\begin{array}{l}\text { flexible working week, } \\
\text { month (S) } \\
\text { horaire flottant (S) }\end{array}$ & choix très libre & individuel & quotidien \\
\hline $5 \mathrm{~d}$. & $\begin{array}{l}\text { horaire variable par } \\
\text { équipe(S) }\end{array}$ & choix assez libre & $\begin{array}{l}\text { individuel } \\
( \pm \text { groupe })\end{array}$ & quotidien \\
\hline 6. & variable working hours & choix totalement libre & individuel & quotidien \\
\hline
\end{tabular}


à des groupes d'employés ou à une seule personne), le temps (l'horaire doit être respecté de façon durable ou peut varier quotidiennement). Ces critères nous ont permis de dresser un tableau comprenant onze types d'horaire que nous avons classés en six catégories suivant le degré de liberté laissé à l'employé (voir : tableau, p. 205). La ligne brisée divisant ce tableau, baptisée «ligne de rupture de la rigidité $»$, sépare le pouvoir décisionnel de la direction d'une entreprise du pouvoir décisionnel de plus en plus grand de l'employé.

Les termes anglais et français qui figurent dans ce tableau ont été retenus suivant certains critères (fréquence, usage, champ sémantique, etc.) qui seront mentionnés au cours de cette étude. Il ne s'agit pas de normes, mais de simples suggestions faites dans le but d'exprimer le plus clairement possible les notions définies.

On notera toutefois que les expressions horaire variable et flexible working hours sont bien implantées dans l'usage et employées par de nombreux auteurs pour désigner les nouvelles formes d'aménagement du temps de travail. C'est pourquoi nous les avons également adoptées comme termes génériques s'appliquant à tous les concepts anglais et français énumérés dans cette étude (sauf horaire fixe, fixed working hours), en plus de les avoir choisies comme les génériques de la cinquième catégorie, qui traite spécifiquement des horaires variables.

\section{DESCRIPTION DES CONCEPTS *}

1. Les heures de travail sont fixes pour tous les salariés et établies par la direction. C'est l'horaire en vigueur actuellement dans la plupart des entreprises. Ex. : Toute la semaine, le personnel travaille de $9 \mathrm{~h}$ à $12 \mathrm{~h}$ et de $13 \mathrm{~h} 30$ à $17 \mathrm{~h}$. Remarque : Le terme horaire fixe désignant ce concept est certes le plus courant et correspond bien au terme anglais fixed working hours.

2. Sur décision de la direction, les employés entrent au travail par groupes à intervalles réguliers, et les premiers arrivés sont également les premiers à quitter le travail.

Ex. : Service $A$ de 8 h 45 à 16 h 45

Service $B$ de $9 \mathrm{~h}$ à $17 \mathrm{~h}$

Service $\mathrm{C}$ de 9 h 30 à 17 h 30

Remarque: En anglais, il existe deux termes pour désigner ce concept, soit staggered day work et staggering of hours. Le premier semble le plus approprié puisqu'il exprime le résultat de l'action plutôt que l'action elle-même.

Quant au français, le terme horaire décalé est souvent employé, mais son synonyme horaire échelonné est préférable parce que «échelonné » signifie «distribué dans le temps, exécuté à intervalles réguliers ». "Décalé », qui a pratiquement le même sens, s'applique toutefois mieux au concept suivant.

* Consulter le tableau de synthèse pour l'étiquetage de chaque concept. 
3a. Sur décision de la direction, il y a déplacement des heures fixes d'arrivée et de départ applicable à tout le personnel. (En fait, ce n'est qu'un déplacement en bloc de " l'horaire fixe » dont il est question au $n^{\circ} 1$.)

Ex. : Du 30 avril au 30 septembre, tout le personnel travaille de 8 h 30 à 16 h 30, tandis que du 30 septembre au 30 avril suivant, il travaille de $9 \mathrm{~h}$ à $17 \mathrm{~h}$.

3b. Même chose qu'en $A$, sauf que c'est le groupe d'employés qui choisit les nouvelles heures de travail. (Ici encore, il s'agit d'un déplacement de «l'horaire fixe », mais choisi par les employés.)

Remarque : La troisième catégorie comprend deux concepts qui ne diffèrent que légèrement l'un de l'autre. C'est pourquoi l'anglais utilise un même terme, staggered working hours, pour désigner chacune de ces notions.

En français, bien qu'il s'agisse d'horaire décalé dans les deux cas, on emploie fréquemment l'expression horaire au choix (qu'on pourrait également appeler " horaire décalé au choix ») pour nommer la deuxième notion puisque le personnel a cette fois-ci la liberté de choisir son horaire collectif et que celui-ci ne lui est plus imposé par la direction.

4a. Parmi les différents horaires fixes établis par la direction, chaque employé effectue un choix durable. Les heures d'arrivée et de départ adoptées deviennent fixes pour lui : chaque jour, il effectue le même nombre d'heures de travail conformément à l'horaire qu'il s'est donné. Cet horaire ne peut être modifié sans l'approbation de la direction.

Ex. : L'employeur établit trois horaires possibles :

de 8 h 30 à 16 h 30 , de 9 h à 17 h et de 9 h 30 à 17 h $30:$ trois employés choisissent le premier, cinq le deuxième et six le troisième.

4b. Même chose qu'en $4 \mathrm{a}$, sauf qu'au lieu de proposer différents horaires à l'employé, la direction le laisse choisir, mais une fois pour toutes, ses heures d'arrivée et de départ, à l'intérieur de deux intervalles de temps donnés (celui du matin et celui du soir).

Ex.: L'employé peut choisir son heure d'arrivée entre 8 h 30 et 9 h 30 , et de départ entre 16 h 30 et 17 h 30 , mais doit s'y astreindre par la suite.

Remarque: La quatrième catégorie, à l'instar de la troisième, comprend deux concepts assez similaires. C'est pour cette raison qu'en pratique l'anglais emploie l'expression fixed flexible working hours pour nommer ces deux notions. Toutefois, par souci de précision, nous avons choisi individual working time pour désigner le concept $4 \mathrm{a}$ car, bien que moins fréquente en anglais, cette expression est plus appropriée puisqu'il s'agit bien d'un choix individuel et non plus collectif (voir tableau).

Le terme horaire individuel a été retenu pour la même raison.

Quant à horaire varié, l'usage tend à imposer cette expression par opposition à «horaire variable» (que nous verrons plus loin). Notons toutefois que « horaire personnalisé » serait également valable puisqu'il illustre bien la légère augmentation de liberté accordée à l'employé par rapport à «horaire individuel » et indique clairement la parenté entre ces deux concepts. 
5a. L'employé établit lui-même, chaque jour, le début et la fin de sa journée (à l'intérieur des plages mobiles) tout en respectant une période fixe (tronc commun) pendant laquelle il doit obligatoirement être à son poste. Il peut y avoir une plage mobile au déjeuner.

Ex. : La présence est obligatoire de $10 \mathrm{~h}$ à $12 \mathrm{~h}$ et de $14 \mathrm{~h}$ à $16 \mathrm{~h}$.

Remarque : La cinquième catégorie traite des horaires variables au sens strict du terme. En effet, l'employé n'est plus soumis à un horaire durable mais peut faire varier ses heures de travail tous les jours. C'est d'ailleurs dans cette catégorie qu'il est question de "plages mobiles », de «plages fixes», de «tronc commun », de « report», de «crédit» ou de « débit» d'heures, etc., qui sont les composantes des horaires variables.

En anglais, flexible working hours (horaire variable), qui regroupe tous les concepts de cette étude (sauf fixed working hours, horaire fixe), est de loin l'appellation la plus courante : mais «pure flexible working hours » est aussi très employée et oppose plus clairement ce type d'horaire à «fixed flexible working hours》 (horaire varié).

L'expression horaire variable tend à devenir la plus usuelle en français international et elle est déjà adoptée par la Régie de la langue française, la Législation française du travail, le Bureau international du travail et le Centre de terminologie d'Ottawa.

5b. Modalité particulière de 5a. (horaire variable) pour laquelle l'horaire comporte $\mathrm{x}$ heures de travail obligatoires par jour; le salarié est donc toujours astreint à une journée «normale » de travail, comportant un nombre d'heures fixe et prédéterminé.

Ex. : La présence est obligatoire de $10 \mathrm{~h}$ à 12 et de $14 \mathrm{~h}$ à $16 \mathrm{~h}$, mais l'employé doit travailler huit heures par jour.

Remarque : L'appellation horaire souple a été retenue pour ce concept parce que l'employé, bien qu'il soit astreint à une journée normale de travail, est tout de même libre de faire varier tous les jours son heure d'arrivée (à l'intérieur d'une plage mobile). Cet horaire offre donc plus de souplesse que les précédents.

5c. Modalité particulière de 5 a. (horaire variable) pour laquelle la période de référence servant au décompte des heures de travail obligatoires est non plus la journée, mais la semaine ou le mois. Il y a souvent possibilité d'effectuer des 《reports 》 d'heures excédentaires («crédits 》) ou d'heures déficitaires (« débits 》).

Ex. : Présence obligatoire de $10 \mathrm{~h}$ à $12 \mathrm{~h}$ et de $14 \mathrm{~h}$ à $16 \mathrm{~h}$ tous les jours, et 40 heures de travail obligatoires par semaine.

Remarque : L'appellation « horaire flottant » est la plus appropriée dans ce cas-ci puisqu'elle décrit le très haut degré de liberté accordé à l'employé. En effet, celui-ci n'est plus astreint à effectuer une journée normale de travail, mais est libre de répartir ses heures de travail sur une base mensuelle ou hebdomadaire.

5d. Autre modalité de 5a. (horaire variable) pour laquelle l'employé doit déterminer ses heures de travail en accord avec les autres membres du groupe dont il fait partie. 
Ex. : La présence peut être obligatoire de $10 \mathrm{~h}$ à $12 \mathrm{~h}$ et de $14 \mathrm{~h}$ à $16 \mathrm{~h}$, et la présence nécessaire de deux membres du groupe peut être de $9 \mathrm{~h}$ à $10 \mathrm{~h}$ et de $14 \mathrm{~h}$ à $15 \mathrm{~h}$.

Remarque : L'équivalent anglais de horaire variable par équipe ne figure pas au tableau parce qu'il n'a pas été trouvé dans les ouvrages anglais consultés.

6. L'employé est totalement libre de travailler quand bon lui semble et souvent d'effectuer le nombre d'heures de travail qui lui convient, pourvu qu'il exécute de façon satisfaisante une tâche qu'on lui a confiée.

Ex. : L'employé travaille de $7 \mathrm{~h}$ à $17 \mathrm{~h}$ le lundi, de $10 \mathrm{~h}$ à $12 \mathrm{~h}$ le mardi, de $9 \mathrm{~h}$ à $15 \mathrm{~h}$ le mercredi, de $8 \mathrm{~h}$ à $16 \mathrm{~h}$ le samedi.

Remarque : En anglais, variable working hours est l'expression la plus courante, de même qu'horaire libre en français.

Attention ! ne jamais traduire «variable working hours » par « horaire variable c'est un faux ami.

\section{VIVIANE FOREST}

MANON ROY

\section{BIBLIOGRAPHIE}

Aménagement ( $L$ ') des temps de travail, l'horaire variable et la semaine comprimée, Département des relations industrielles (Úniversité Laval), Les Presses de l'Université Laval, Québec, 1974, 337 p.

BAUDRAZ, J.-F., l'Horaire variable de travail, Les Éditions d'Organisation, Paris, 1973, 135 p.

BAUM, S.J. et W.M. YOUNG, A Pratical Guide to Flexible Working Hours, Londres, Kogan Page Limited, 1973, 186 p.

BOLTON, J. H., Flexible Working Hours, Anbar Publications Lt., Londres, 1971.

BOUCHER, N.-C., "Les horaires de travail en pleine évolution ", Commerce, avril et mai 1974.

CONFERENCE BOARD IN CANADA, The Altered Work Week, Ottawa, novembre 1973.

BOYER, Marc, le Tourisme, Éditions du Seuil, Paris, 1972, 261 p.

CHAMBRE DE COMMERCE DU DISTRICT DE MONTREAL, la Semaine comprimée et les horaires flexibles, La Maison du Commerce, Montréal, 23 avril 1973.

ELBING, A. O., J. R. M. GADON, H. GORDON, Flexible Working Hours, Harvard Business Review, janvier-février 1974.

EVANS, A., Temps et vie de travail, Organisation de coopération et de développement économique (OCDE), Paris, 1973.

GROSSIN, W., le Travail et le temps, Éditions Anthropos, Paris, 1969.

Horaire variable ou libre $(L '), \mathrm{La}$ Documentation française, Rapport du groupe d'étude réuni à la demande du Premier Ministre, 28 avril 1972, Paris, 1972, 81 p.

Horaires de travail (Les), Régie de la langue française, $2^{\circ}$ éd., Québec, Gouvernement du Québec, mars 1975.

Image et Formation, Informatech France-Québec, s.d., s.1., 192 p. + supplément.

KAPP, B., D. PROUST, les Horaires libres, Chotard et Associés éditeurs, Paris, 1973, 315 p. 
MARSH, A. I. et E. C. EVANS, The Dictionary of Industrial Relations, Hutchison of London, Londres, $1973,415 \mathrm{p}$.

OCDE, Direction de la main-d'œuvre et des affaires sociales, l'Aménagement du temps de travail, Paris, 1973.

POLYSCOPE, (Le), Journal de l'Association des étudiants de Polytechnique, "Humaniser le travail », vol. IX, $\mathrm{n}^{\circ} 14,19$ janvier 1976.

Production et gestion, $\mathrm{n}^{\circ} 253$, septembre 1973, p. 16.

ROBERT, Paul, le Petit Robert, Société du Nouveau Littré, Paris, 1967.

ROBERTS, H.S., Roberts' Dictionary of Industrial Relations, The Bureau of National Affairs Inc., Washington (D. C.), 1971, 599 p.

The Random House College Dictionary, New York, Random House Inc., 1972.

WADE, M., Flexible Working Hours in Practice, Epping, Essex, Gower Press Ltd., 1973, 112 p. 